\title{
Measuring the Structure of Road Networks
}

\author{
Feng Xie, David Levinson \\ Department of Civil Engineering, University of Minnesota, Minneapolis, MN
}

\begin{abstract}
Spatial networks display both topologic and geometric variations in their structure. This study investigates the measurement of a road network structure. Existing measures of heterogeneity, connectivity, accessibility, and interconnectivity are reviewed and three supplemental measures are proposed, including measures of entropy, connection patterns, and continuity. The proposed measures were applied to 16 test networks, which were derived from four idealized base networks: $90^{\circ}, 45^{\circ}, 30^{\circ}$, and completely connected. The results show that the differentiated structures of road networks can be evaluated by the measure of entropy; predefined connection patterns of arterial roads can be identified and quantified by the measures of ringness, webness, beltness, circuitness, and treeness. A measure of continuity evaluates the quality of a network from the perspective of travelers. Proposed measures could be used to describe the structural attributes of complicated road networks quantitatively, to compare different network structures, and to explore the structural evolution of networks in the spatial and temporal context. These measures can find application in urban planning and transportation practice.
\end{abstract}

\section{Introduction}

Networks, which can be embodied as a set of nodes representing spatial locations and a set of links representing connections, possess many different structural properties, displaying both topological and geometric variations. The arrangement and connectivity of nodes and links of a network is referred to as its topology. At a higher level of complexity, geometric attributes such as spacing, shape, orientation, density, and geometric patterns may be introduced.

The long-standing interest in measuring the spatial structure of road networks has been driven by the inherent impact of network structure on the performance of transportation systems, as well as its subsequent effects on land use and urban form (Mohring 1961; Gauthier 1966; Marshall 2005). Quantifiable indicators can abstract the properties of a complicated network structure and could be helpful in

Correspondence: Feng Xie, Department of Civil Engineering, University of Minnesota, 500 Pillsbury Drive SE, Minneapolis, MN 55455

e-mail: xiex0055@umn.edu

Submitted: August 26, 2005. Revised version accepted: October 19, 2006. 
exploring the structural evolution of these networks in a spatial and temporal context.

Early work on measuring the structure of transportation networks dates back to the 1960s, when geographers and transportation researchers focused almost exclusively on topologic measures using graph-theoretic network analysis, constrained by limited data, computational power, and modeling techniques (Garrison 1960; Garrison and Marble 1962; Kansky 1963; Hargett and Chorley 1969). With the more widespread availability of travel demand models, researchers started to explore how traffic flows and travel pattern are affected by various geometric network structures (Newell 1980; Vaughan 1987). Some empirical studies analyzed specific connection patterns of roads, especially urban highways, both qualitatively and quantitatively (Payne-Maxie Consultants 1980; Taylor 1995). In recent years, network research has shifted its focus from simple topologic and geometric properties to large-scale statistical properties of complex (and less spatially constrained or nonspatial) networks (Albert, Jeong, and Barabasi 1999; Barabasi 2002; Barabasi and Bonabeau 2003; Newman 2003). Some of these studies used as examples large-scale transportation networks such as the national airline system and the national highway network.

This study reviews previous research on transportation network structures and proposes three complementary structural measures of heterogeneity, connection pattern, and continuity based on the characteristics of roads.

The functional or operational differentiation of roads in a network with regard to their relative importance to the network is referred to as network heterogeneity, which is evaluated in this study by a collective statistical measure of entropy.

This study explores the geometric pattern of arterials, which connect contiguously in a network, as an outstanding feature of most road networks. Predefined connection patterns are identified and their respective significance is measured.

Network structure shapes traffic flows on a network. The desired properties of network structure perceived by travelers in their travel such as clarity, contiguity, and comfort are important indicators to the quality of network design and urban transportation planning. The third measure proposed in this study is developed based on travel demand models to evaluate the aggregate continuity/discontinuity experienced by travelers when moving on a given network that consists of different classes of roads.

The next three sections review the literature and present three proposed measures. Then, these measures are applied to 16 test networks and the results are analyzed. The concluding part summarizes the findings and suggests the potential application of the proposed measures.

\section{Heterogeneity}

Heterogeneity is a common feature of many complex networks. Barabasi and Bonabeau (2003) found that sites on the Web form a network with unique 
mathematical properties: the probability $\left(p_{k}\right)$ that any node (site) was connected to $k$ other nodes was proportional to $1 / k^{\alpha}$, that is,

$$
p_{k} \propto \frac{1}{k^{\alpha}}
$$

where $k$ is the degree of the node, that is, the number of links connected to that node; $\alpha$ is some constant exponent.

The distribution of node degrees defined by equation (1) is called the "powerlaw degree distribution" (Newman 2003). Complex networks with power-law distributions are referred to as "scale-free networks" in a study of the World Wide Web (Albert, Jeong, and Barabasi 1999) and have been observed in a host of other networks, notably including citation networks (Price 1965), metabolic networks (Jeong et al. 2000), and the network of human sexual contacts (Liljeros et al. 2001).

Although scale-free networks are now recognized, the power law is not a universal rule. All the networks in which most nodes have approximately the same number of links are referred to as "random networks," in contrast to scale-free networks that are subject to a power law. A road network is one of many prominent random networks that exist in the real world. Barabasi and Bonabeau (2003) state that "there might be steep cost addition of each link to a given node" that "could prevent certain networks from becoming scale-free." In the case of an urban road network, constructing and operating an intersection (either a grade-separated interchange or a surface intersection with signals) is costly, and the cost increases exponentially with the number of intersecting links. Thus, a node of intersection tends to connect only to a few adjacent nodes. Limited road capacity is another factor that prevents a road network from becoming scale-free. As Barabasi pointed out, "congestion along specific links is major consideration" for transportation because "too much traffic on a particular link can cause the potential failure of this link and other links." In addition, due to the vast investment in right of way and infrastructure for limited-access long-distance roads, a node tends to connect to other nodes with limited distances.

These explanations, however, fail to recognize the link-centric nature of road networks. In fact, while Barabasi deals in a node-centric world, links are the active elements in surface transportation infrastructure. The limited number of connections of nodes and limited capacity and lengths of links do not mean that urban roads are homogenous. Instead, we would argue that heterogeneity exists in most road networks, considering the differentiated functional properties and operational performance of urban roads, and that this heterogeneity (hierarchy) would exist without a prespecified design (Yerra and Levinson 2005; Levinson and Yerra 2006).

"Functional classification" is the process by which streets and highways are grouped into classes according to the character of service they are intended to provide (Federal Highway Administration 1997). The road network plays a dual role in providing both access to property and travel mobility. Local streets emphasize the land access function, arterial roads emphasize a high level of mobility for 
through movement, and collectors offer a compromise between both functions. Arterial roads (including freeways, major highways, and undivided arterials) are essential in an urban transportation system with regard to the mobility. In Minnesota, for example, there are approximately 19,300 km (12,000 miles) of Interstates and state highways (9\% of the total road length) as of 2005, which account for about $60 \%$ of the total 87 billion yearly vehicle-kilometers (54 billion vehiclemiles) traveled in this state (Minnesota Department of Transportation 2005). Arterials are usually designed with higher free flow speeds, higher capacities, and longer uninterrupted distances.

Roads also have different operational performance, in terms of their levels of service (LOSs). LOS incorporates elements such as riding comfort and freedom from speed changes, but the most basic is operating speed or trip travel time. This kind of differentiation can be called an "operational classification."

The functional classifications of roads in design, combined with the differentiation of road performance in operation, make an urban road network heterogeneous.

In a scale-free network, the importance of a node is indicated by the degree of that node (Newman 2003) and using each node's degree as a proxy for its importance, the heterogeneity of a complex network can be further statistically quantified (Sole and Valverde 2004; Trusina et al. 2004). Without taking into account link properties, however, few of these measures can be used for urban road networks. This section introduces a statistically collective measure of entropy $(H)$ to evaluate the link-based heterogeneity of road networks.

The concept of entropy was initially proposed by Shannon (1948) in his landmark article, "A Mathematical Theory of Communication," to measure information uncertainty:

$$
H(X)=-\sum_{i=1}^{m} p_{i} \log _{2}\left(p_{i}\right)
$$

where $m$ is the number of subsets in the system $X$ and $p_{i}$ is the proportion of agents in the $i$ th subset. The entropy value of a homogenous group is zero and a higher entropy value indicates more heterogeneity. Entropy has also been widely introduced to measure the heterogeneity of complex networks (Balch 2000; Ben-Naim, Frauenfelder, and Toroczkai 2004).

If individual links of a road network are considered as a collection of agents, they can be grouped into subsets based on different road properties such as functional type, traffic volume, or LOS. For example, the 20,380 links in the Minneapolis-St. Paul Metropolitan Area Planning Network are in practice grouped into six different LOSs from LOS A to $\mathrm{F}$ or nine different functional types including divided freeways, undivided freeways, ramps, collectors, and so on. The proportion of each subset is calculated as the frequency of links in this subset over the total number of links, and then proportions are aggregated into the entropy measure in 
equation (2). In an extreme case, all the links are grouped into one subset. Without losing generality, if we suppose

$$
p_{i}= \begin{cases}1 & i=1 \\ 0 & i>1\end{cases}
$$

the entropy measure is equal to 0 , representing a homogenous network with regard to a specific road property. A positive entropy measure indicates that there exists heterogeneity in a network with more than one group of links. A larger entropy measure indicates a greater heterogeneity of the network.

\section{Connection pattern}

The connection and arrangement of a road network is usually abstracted in network analysis as a directed planar graph $\boldsymbol{G}=\{\boldsymbol{V}, \boldsymbol{E}\}$, where $\boldsymbol{V}$ is a collection of nodes (vertices) connected by directional links (edges) $\boldsymbol{E}$ (links are directional when a link from node $R$ to node $S$ is distinct from a link from $S$ to $R$ ). Physically, a two-way road consists of two adjacent and opposite one-directional links. A network that consists of only two-way links can be simplified as an undirected network. This study examines only undirected networks for simplicity.

A number of topological measures of network structure have been developed based on elementary concepts of graph theory. Four indices, including Cyclomatic number, $\alpha$ index, $\beta$ index, and $\gamma$ index, are all defined on the basis of three basic parameters of network topology, that is, the number of edges (road segments) (e), the number of vertices (nodes) $(v)$, including road intersections, travel origins and destinations, and the number of maximally connected components $(g)$, which is explained as follows: a planar network may be unconnected but may consist of connected pieces, which are called "maximally connected components," or "connected components." Given a network $\boldsymbol{G}=\{\boldsymbol{V}, \boldsymbol{E}\}$, its subgraph $\boldsymbol{S}=\left\{\boldsymbol{V}^{\prime}, \boldsymbol{E}^{\prime}\right\}$ is a maximally connected component if all vertices $\left(\boldsymbol{V}^{\prime}\right)$ of $\boldsymbol{S}$ are connected by edges in $\left\{\boldsymbol{E}^{\prime}\right\}$, and no vertex can be added to $\boldsymbol{S}$ so that $\boldsymbol{S}$ will still be connected. The total number of connected components $g$ in a network can be counted using graph algorithms (Gibbons 1985).

The cyclomatic number indicates the number of circuits in a network. The $\alpha$ index is the ratio between the actual number of circuits in the network and the maximum number of circuits; the $\beta$ index is the ratio between the number of links and the number of nodes; and the $\gamma$ index compares the actual number of links with the maximum number of possible links in the network.

These indices can estimate the multiplicity of links in a road network and can also form some useful common yardsticks for comparison between networks. Values for the $\alpha$ index and $\gamma$ index range from 0 to 1 . A higher value for each of the four measures represents a more connected network.

A series of matrices for direct connections, accessibility, the Shimbel distance, and valued graph are also developed to examine the internal structure of networks 
(Taaffe, Gauthier, and O'Kelly 1996). These matrices can be used to conduct network analysis that cannot be effectively treated by single-number full-network measures discussed above.

More details on these measures are summarized by Hargett and Chorley (1969), Taaffe, Gauthier, and O'Kelly (1996), and Rodrigue (2004). These measures have been used to explore the topology of transportation networks since the early 1960s. Garrison (1960) measured the connectivity of the Interstate Highway System. Kansky (1963) proposed 14 indices to measure the topological characteristics of transportation networks. Dill (2004) measured the network connectivity for bicycling and walking in the Portland, Oregon region.

While these descriptive measures are useful, they are incomplete as they disregard the distance and orientation of links. Among all the geometric attributes of urban road networks, connection pattern has been the focus of attention (Hargett and Chorley 1969; Hanson 1986; Vaughan 1987; Taaffe, Gauthier, and O'Kelly 1996; Marshall 2005) for its fundamental impact on travel behavior, distribution of homes and workplaces, land use, and urban form. Specifically, typical connection patterns such as beltway and hub-and-spoke in transportation systems have been studied for years.

Originally conceived as a means of diverting through traffic away from congested central-city areas, beltways have become integral parts of the intrametropolitan highway system. The U.S. Department of Transportation performed a study to examine the impacts of circumferential limited-access highways (beltways) on the land use of American cities (Hanson 1986). A statistical analysis was performed on a set of 27 beltway cities (such as Washington, DC) and 27 nonbeltway cities (such as Pittsburgh, Pennsylvania) in the United States, but no significant land-use impacts were found from the existence, relative location, or length of beltways (Payne-Maxie Consultants 1980).

The hub-and-spoke network is another typical connection pattern in a variety of contemporary transportation systems such as air and freight transportation, express delivery systems, container shipping, and military logistics system. Taaffe, Gauthier, and O'Kelly (1996) examined the rationale for hub-and-spoke networks using basic transportation and economic geography. They discussed some actual hub-and-spoke examples in air transportation and also dealt with applications of hub-and-spoke to surface transportation in the discussion of intermodalism. The hub-and-spoke system became the norm for most major airlines (the so-called "network carriers") in the United States after airline deregulation in 1978 (Morrison and Winston 1989). Bryan and O'Kelly (1999) reviewed the advances in analytical hub location problem for the airline hub-and-spoke networks. Taylor, Harit, and English (2005) addressed configuration and operational concerns on hub-andspoke networks in trucking.

Despite the contributions of these studies, few of them have investigated the patterns of roads quantitatively. Connection patterns of road networks remain poorly understood by engineers and urban planners. As Marshall (2005) pointed 
out, although the connection patterns of streets and roads play a profound role in the city form as a physical presence and as a land use, designers have to describe their desired patterns of roads subjectively and abstractly, due to the lack of their explicit measurement.

This section defines four typical connection patterns in road networks, develops an algorithm to identify inherent patterns of a given network, and proposes quantitative measures to evaluate the significance of each specific connection type.

There are two basic structures for planar transportation networks: branching networks and circuit networks (Hargett and Chorley 1969). Circuit networks are regional networks structured with closed circuits, where a circuit is defined as a closed path (with no less than three links) that begins and ends at the same vertex. Branching networks are distinguished by their tree like structures, which consist of sets of connected lines without any complete circuits. Specifically, a graph containing no cycles is called a forest and a connected forest is called a tree. Typical connection patterns emergent in circuit or branching transportation networks include ring, web, star, and hub-and-spoke. Fig. 1 illustrates simple examples of these connection patterns and their graph-theoretic definitions follow.

In a branching network with its Cyclomatic number $(u)$ equal to zero, a hub is usually defined as a node with more than two connections. A branching network with a single hub is defined as a star while a branching network with multiple hubs is defined as a hub-and-spoke system.

When the Cyclomatic number is larger than zero, there is at least one circuit in the network. In order to further define the connection types in such a network, the concepts of "bridge" (or "cut edge") and "articulation points" are also introduced according to Gibbons (1985).

An edge in a connected graph is a bridge if deleting it would create a disconnected graph. An articulation point of a connected graph is a vertex whose removal disconnects the graph. A subnetwork is called a block if it contains no articulation points. Note that the blocks of a graph with one or more articulation points can be identified by disconnecting the graph at each articulation point in turn in such a way that each block retains a copy of the articulation point.

A circuit block is defined in this study as a block that contains at least one circuit and contains neither bridges nor articulation points. If a circuit block contains only one circuit, it is defined as a ring; if it contains more than one circuit, it is defined as a web.

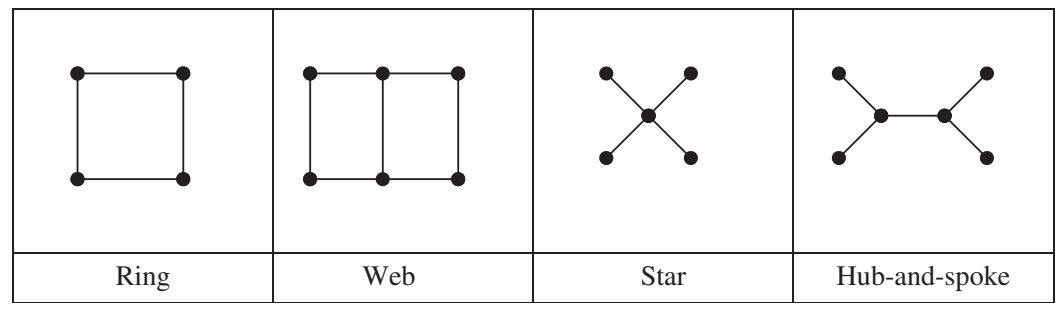

Figure 1. Examples of connection patterns. 
Considering arterials as a subnetwork of a road network, this study examines the connection patterns of arterial roads in particular. The reason is twofold. First, according to the functional classification of roads, arterials play an essential role in travel mobility, serving as the backbone of a road system. The topology of arterials may have a more direct and essential impact on the overall travel mobility of a road network than that of local streets; second, the arterial network is smaller than the whole road network and thus demonstrates clearer patterns that are easier to define and identify. In fact, all the aforementioned studies on road connection patterns examine highway systems only.

Marshall (2005) has observed an outstanding feature that the national road network possesses: strategic routes all connect contiguously. Marshall refers to this property as "arteriality." An arterial network itself may be unconnected and consists of pieces of connected components $(g)$, as defined above. The primary component is defined as the connected component that comprises the largest length of arterials. This study evaluates the relative size of the primary component by comparing the length of the primary component with the total length of arterials as a ratio $\phi_{\text {prim. }}$. The values $g$ and $\phi_{\text {prim }}$ indicate how dispersed arterials are distributed in a road network and thus measure the arteriality of the network. A large $g$ together with a small $\phi_{\text {prim }}$ implies that arterials are scattered without connecting into continuous routes and thus represents a road network of low arteriality. A small $g$ and a large $\phi_{\text {prim }}$ on the other hand, indicate a road network of high arteriality. Arterials in most real road networks have only one connected component with $g$ equal to 1 and $\phi_{\text {prim }}$ equal to 1.0 .

Fig. 2 illustrates how circuit blocks can be identified algorithmically from a connected arterial network, based on which the structural elements of branch, ring, circuit, and beltway can be identified and their relative significance can be measured. A circuit block has the following properties according to graph theory:

(1) As a circuit block contains no bridge or articulation points, it will remain connected after deleting any node or link.

(2) There are at least two paths between any pair of nodes without any common nodes between paths, except the origin and the destination, which ensures "multiple paths between all origins and destinations, and at least two of which share no links" in a web (Levinson 2002).

(3) A link on a circuit must belong to one of the circuit blocks; a link on a circuit block can belong to either a ring or a web. This study measures the "ringness" and "webness" of a network by ratios:

$$
\begin{aligned}
& \phi_{\text {ring }}=\frac{\text { Total length of arterials on rings }}{\text { Total length of arterials }} \\
& \phi_{\text {web }}=\frac{\text { Total length of arterials on webs }}{\text { Total length of arterials }}
\end{aligned}
$$




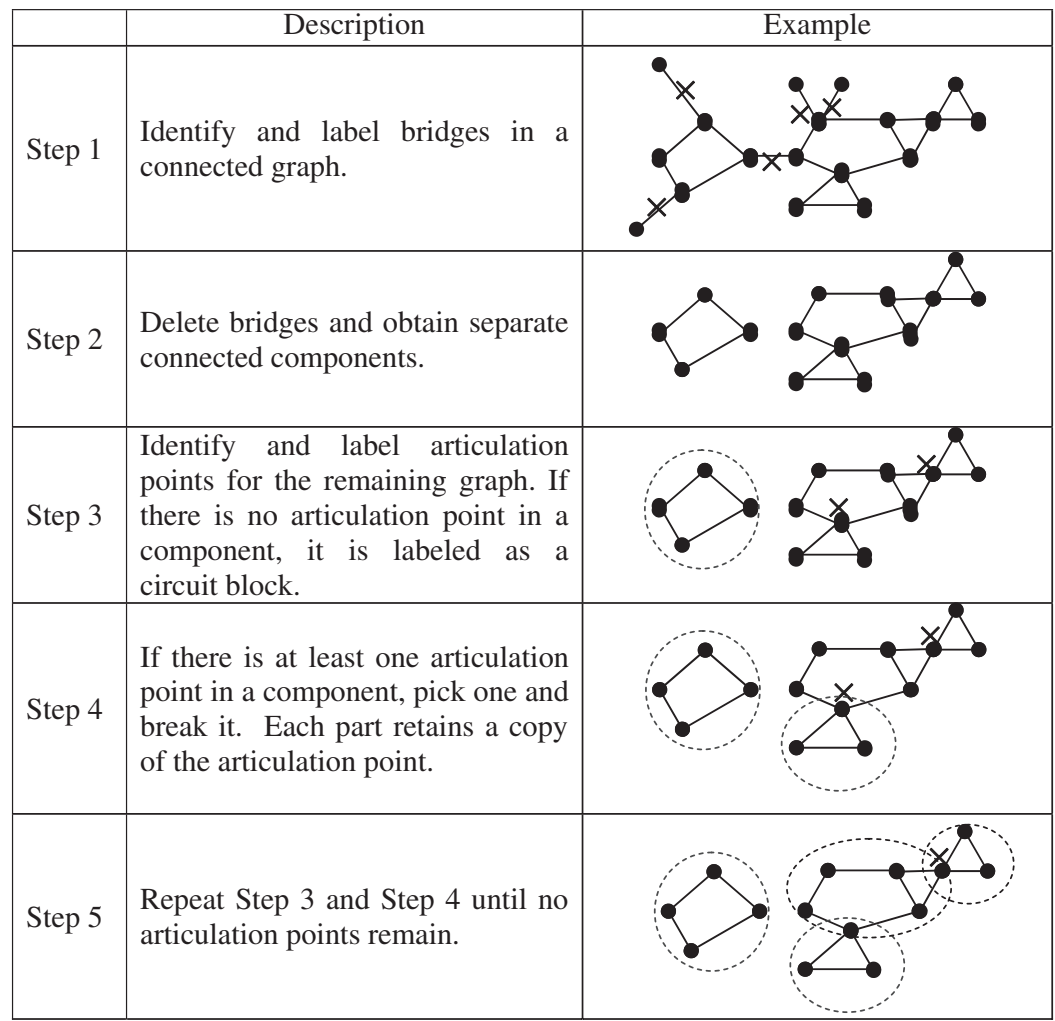

Figure 2. Identification of circuit blocks in a connected graph.

The concepts of "circuitness" and "treeness" can be further defined as:

$$
\begin{gathered}
\phi_{\text {circuit }}=\phi_{\text {ring }}+\phi_{\text {web }} \\
\phi_{\text {tree }}=1-\phi_{\text {circuit }}
\end{gathered}
$$

These ratios range from 0.0 to 1.0 , indicating to what extent arterials are connected as circuits or trees. A high ratio of treeness indicates a branching structure while a high ratio of circuitness indicates a circuit network. These definitions and measures provide a consistent and computable way to examine typical topologies for the arterial network based on digitized road networks.

(4) The series of connected links on the envelope of a circuit block forms the largest circuit contained by this block. The beltway study for the U.S. Department of Transportation (Payne-Maxie Consultants 1980) displays the highway networks of eight typical beltway cities in the United States. As shown in Fig. 3, a metropolitan highway network usually contains a major web block embracing the downtown area and a beltway located on the 


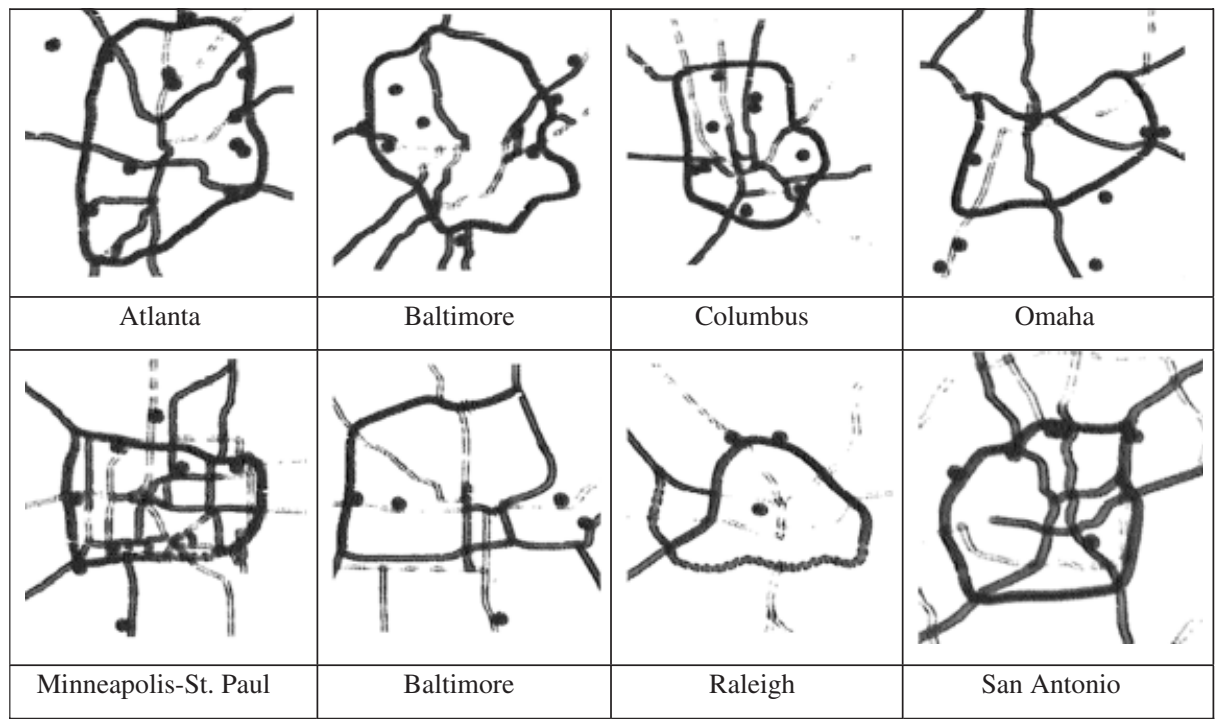

Data Source: Payne-Maxie Consultants (1980)

*The bold lines represent highways; the dots represent regional shopping centers

Figure 3. The 1976-1980 highway networks of eight beltway cities.

border of the web block. The beltway study, however, did not define a beltway accurately or point out how to identify a beltway in a developed urban road network. In this study, we define a beltway in a road network where one dominant circuit block exists. If the dominant block is a ring, the ring is identified as the beltway; if the dominant block is a web, we define the beltway as the envelope of the circuit block. Although the identification of a beltway in a real road network may be subject to other factors, such as designations of arterials, convexity of the belt, and distance from the central business district (CBD), our definition will be a good approximation. Based on our definition of beltway, a unique beltway can be identified algorithmically for any road network with a dominant circuit block, and its length, coverage area, number of interchanges, and traffic volume can be easily estimated. This study proposes a simple ratio of "beltness" to indicate the significance of beltway:

$$
\phi_{\text {belt }}=\frac{\text { Length of the beltway }}{\text { Total length of arterials }}
$$

An urban arterial network may have multiple concentric beltways around its $\mathrm{CBD}(\mathrm{s})$. An inner beltway can be identified by breaking its outer beltway and repeating the above procedures. Specifically, we remove the nodes on the envelope of the dominant circuit block that we have identified as the outer beltway, find the inner dominant circuit block, if any, for the remaining part of the block, and identify the envelope of this circuit block as the inner 
beltway. These steps can be repeated until no dominant circuit block is found. The beltness of each beltway can be calculated. To distinguish, we mark the beltness of the first beltway from the outside as $\phi_{\text {belt, }}^{1}$ that of the second inner beltway as $\phi_{\text {belt, }}^{2}$ and so on.

\section{Continuity}

All the aforementioned measures examine the structure of road infrastructure network while disregarding traffic flow on the network. In an urban transportation system, however, traffic flow and network structure are mutually affected. Daily travel behaviors aggregate into traffic flow on the network, while in turn the human movement on the network transforms network structure in the long run. During this process, there are always inconsistencies between the desired properties that a network structure is expected to possess in design and the quality of travel that is actually perceived by travelers in using the network, such as legibility, convenience, comfortableness, and consistency.

As traditional measures of accessibility account for the impedance between places only in terms of the distance or time of travel, interconnectivity becomes a property of transportation networks that has been under active investigation, assuming inconvenience is associated with transferring between different levels of roads. Lee and Lee (1998) examined connection between transit lines. Rietveld (1995, 1997) discussed both interconnectivity between multiple transportation modes and interconnectivity in unimodal networks based on different service levels, for instance, high speed, few stops versus lower speeds, many stops. Zhang (2006) estimates user resistance to discontinuity in route selection empirically.

This section proposes a measure of continuity that examines travelers' perceptions of the interconnectivity in an urban road network. As pointed out by Rietveld (1995), "the quality of transport networks does not only depend on the features of the links, but also on the way the links are connected." When travelers move on a road network that consists of different classes of links, they usually have to transfer from one class of roads to another at the intersections or interchanges along their paths (routes). During this process, discontinuity may be perceived due to the inconvenience associated with transferring between different classes of roads. Fig. 4 gives three possible travel patterns on different two-level networks. Part (1) presents a typical travel pattern on an urban road network. Travelers access a highway (arterial) via a local street (collector), complete the largest part of their trips on highways, and return to a local street to reach their destinations. Part (2) illustrates a possible travel pattern on a hypothetical network on which roads of different hierarchies are randomly distributed. Travelers have to frequently transfer between roads of different hierarchies, and in this case they would travel less than they do on the previous network. Part (3) exemplifies the travel pattern on a rural road network. As only local streets exist, motorists would make even shorter trips. As can be seen, a moderate frequency of transfers on roads of different hierarchies helps travelers 

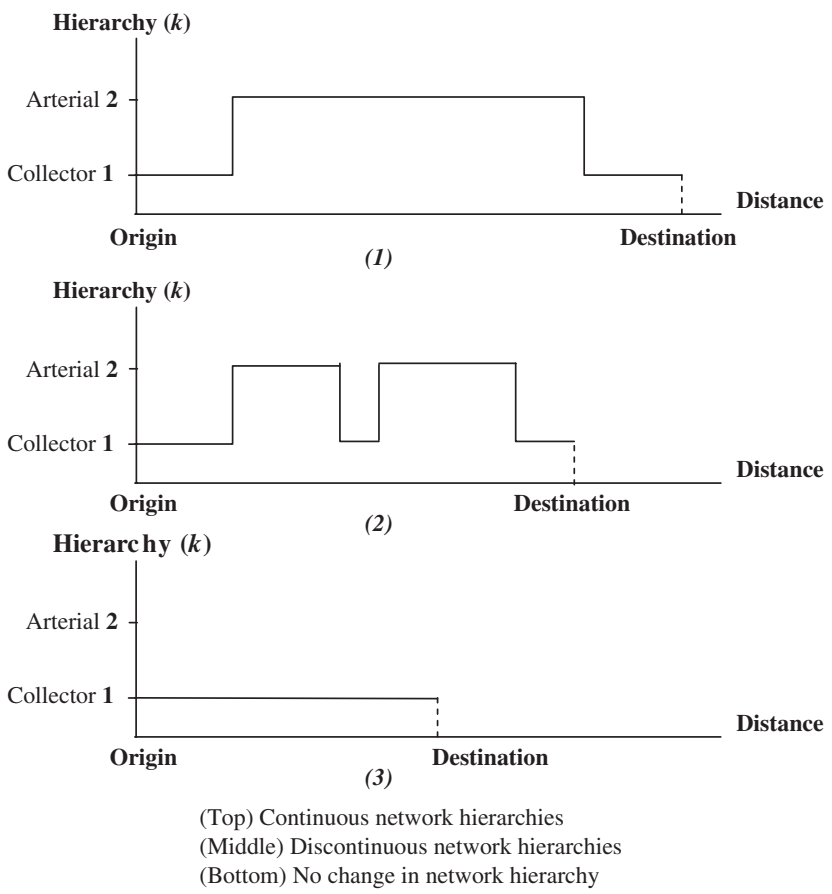

Figure 4. Example travel patterns on two-hierarchy networks.

access arterial roads and choose the most efficient route, while too many transfers may raise the discontinuity of vehicle speed and thus decrease people's willingness to travel. "Continuity" and its complement "discontinuity" are introduced to capture the inconvenience associated with transferring between different classes of roads. In a sense, the measure of continuity can be regarded as a mixed evaluation of both heterogeneity and interconnectivity in a network from the perspective of travelers.

As we assign all the origin-destination $(\mathrm{O}-\mathrm{D})$ traffic on the shortest path between the origin and the destination, the discontinuity of a trip can be measured as the changes of link hierarchy along the shortest path. If a traveler moves from the upstream link $a$ of hierarchy $k_{1}$ to the downstream link $b$ of hierarchy $k_{2}$, the discontinuity of this movement is measured as:

$$
y_{a}=\left|k_{1}-k_{2}\right|
$$

Travel demand models are used to predict the traffic flow on each route connecting any $\mathrm{O}-\mathrm{D}$ pair. For simplicity of illustration, we assume that all the traffic between a certain $\mathrm{O}-\mathrm{D}$ pair is allocated to the shortest path according to an all-ornothing assignment (Ortuzar and Willumsen 2001). The improved computing power enables us to trace the journey of travelers along each shortest path and measure their perceptions on the continuity of their travel in the network. 
Thus, the discontinuity of the trip along the shortest path $\{P\}$ can be measured as

$$
Y(P)=\sum_{a \in\{P\}} y_{a}
$$

The discontinuity of a road network can be measured as

$$
Y=\frac{\sum_{\text {all }(R, S)} Y\left(\left\{P_{R S}\right\}\right) \times q_{R S}}{\sum_{\text {all }(R, S)} I\left(P_{R S}\right) \times q_{R S}}
$$

where $P_{R S}$ is the shortest path between a given O-D node pair $(R, S), q_{R S}$ is the number of trips between the origin and the destination, and $I(P)$ is the length of the shortest path.

\section{Experiments}

This study introduces four idealized networks with simple geometrical properties as base networks and derives 12 other networks by removing links from the base networks and specifying hierarchy levels for the remaining links. The base networks are characterized by which directions that one can travel from any point in the network, and they are referred to in this study as the $90^{\circ}, 45^{\circ}, 30^{\circ}$, and completely connected (or complete) networks, respectively.

A $90^{\circ}$ network is a network consisting of square grids. We call it the $90^{\circ}$ network because travelers can only make a turn of $90^{\circ}$ or a multiple of $90^{\circ}$ at any node of such a network. Similarly, a $45^{\circ}$ network is a network in which the included angle of two intersecting links can be either $45^{\circ}$ or multiples of $45^{\circ}$. A $30^{\circ}$ network has the same topology with the hexagon landscape developed consistent with the transportation principle of central place theory (King 1985), in which the included angle of two intersecting links can be $30^{\circ}$ or its multiples. A complete network is developed by connecting every pair of the intersection nodes of a grid network and dividing direct connections into shorter links where they intersect or overlap. Theoretically, the turning directions included in a complete network range from $0^{\circ}$ to $360^{\circ}$, depending on the size of the original grid network. As all these networks are developed based on the square grid network, their size can be indicated by the number of nodes along each side of the original square grid. For example, a $4 \times 4$ complete network is a network developed on a $4 \times 4$ grid.

Fig. 5 displays four base networks, $15 \times 1590^{\circ}$ network (A0), $15 \times 1545^{\circ}$ network (B0), and $15 \times 1530^{\circ}$ network (C0), and $4 \times 4$ complete network (D0), as well as 12 network structures derived from them (three for each). As can be seen, links have been specified as five different hierarchies. The boldness and grayness of a link indicates its hierarchy level and a bolder and darker link represents a road of a higher hierarchy. This study examines these 16 networks with proposed structrual measures. 


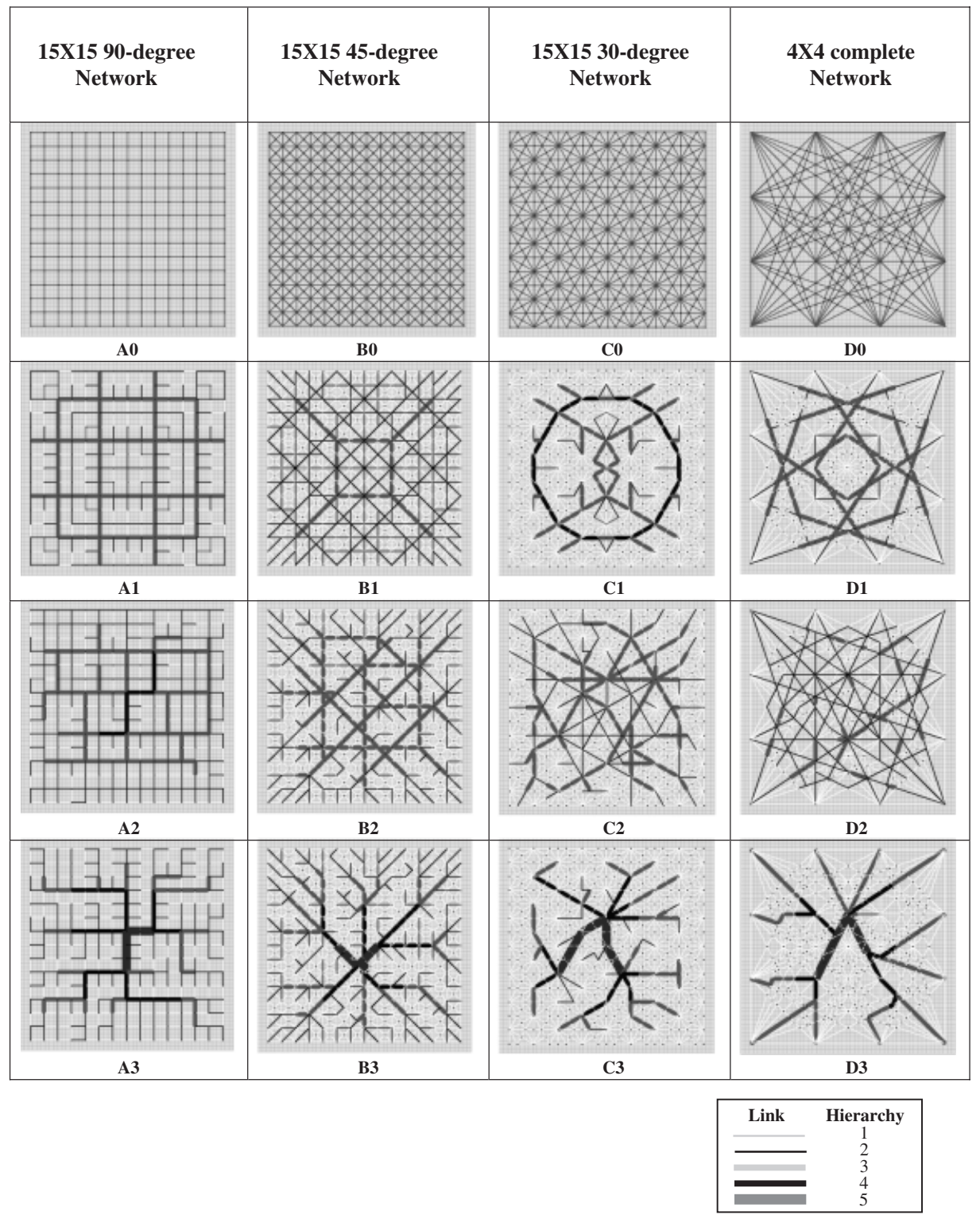

Figure 5. Sixteen test networks.

In addition, to incorporate land-use patterns into our measurement, we assume each network is located in the center of a land-use grid. In this study, a uniform land-use distribution on a square land use layer (2500 land-use cells) is prespecified, with each land-use cell generating and attracting 10 trips of a single mode. All the trips are allocated to the nearest node that is connected to the network and, as mentioned before, all the traffic between any pair of nodes is assigned to the 
shortest path. The travel demand model used in this study follows Yerra and Levinson (2005), including the steps of the shortest path finding, gravity-based trip distribution, and traffic assignment.

\section{Results}

With the prespecified five categories of links, we calculated the entropies of test networks. With the assumption that roads of Level 3 and higher represent arterials, we examined links of Levels 3, 4, and 5 in particular, measuring the ringness, webness, and treeness of arterial networks, and identifying their connection types. We also calculated the discontinuities of test networks with the prespecified landuse layer and simplified travel demand models. Table 1 presents the basic structural properties for the test networks.

As shown in Table 1, the first column lists the total length of links for each test network. As each network occupies the same area of land-use territory, this measure can indicate the relative density of road infrastructure. As can be seen, the network B0 is the most intensively developed network, which has the longest distance of roads, as well as the largest number of links and nodes among test networks. The topological measures for the arterial subnetwork are not available for A0, B0, C0, and D0 because these networks consist only of links on Level 1 (collectors). The fourth column lists the number of connected components in the arterial subnetwork $(g)$, and the fifth column lists the length ratio of the primary component $\left(\phi_{\text {prim }}\right)$. Together, they indicate the arteriality of each road network. As can be seen, network D2 is the only network containing 18 scattered pieces of arterials, while all the others have only one connected network of arterials. The number of circuits $(u)$ indicates whether a network is a circuit network or a branching network. More accurate measures like circuitness and treeness will be discussed later. Note that networks A1, A2, B1, B2, C1, and D1 each contain a dominant circuit block, and so their beltways can be identified and their measures of beltness can be calculated based on our definition. Some of these beltway networks may contain multiple beltways.

Table 2 summarizes the results of proposed structural measures for these networks.

The four base networks are displayed, respectively, in A0, B0, C0, and D0. Again, as these four networks do not contain any arterial, both the entropy measure and the discontinuity measure of each network are equal to 0.0. Furthermore, the measures of ringness, webness, and treeness for these networks are not available in Table 2 .

Note that base networks are all symmetric structures. Networks A1, B1, C1, and D1 are also symmetric structures derived, respectively, from the four based networks, and they are all characterized by significant beltways in the central area of landscape. Network B1 contains a ring in the center as well as four radiating arterial links extending to the corners. Networks A1, C1, and D1 each contains a dominant web block, and beltways can be identified approximately along the envelopes of 


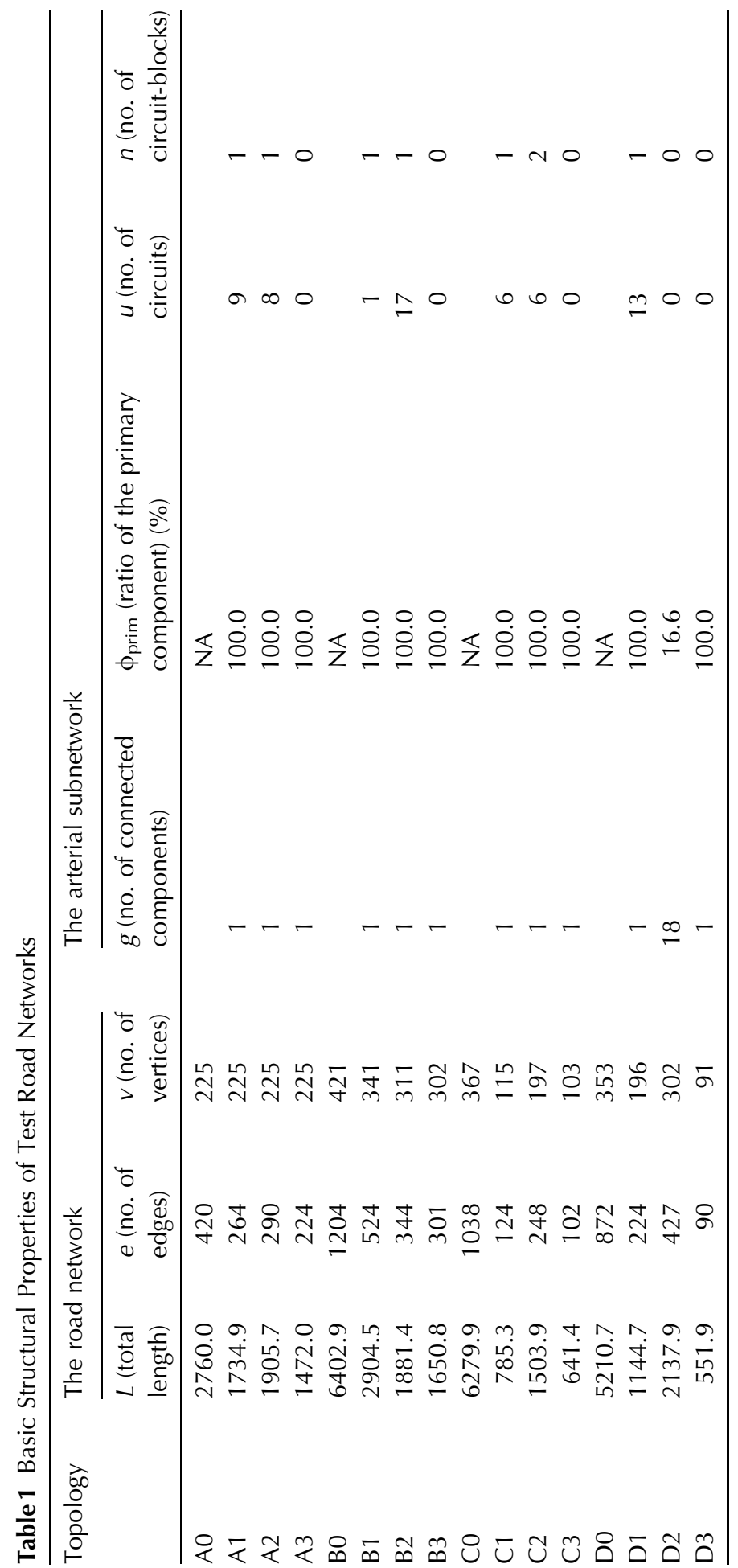


these web blocks. All these networks have relatively low measures of treeness, and relatively high measures of circuitness.

Networks A2, B2, C2, and D2 represent relatively complicated asymmetric structures derived from the base networks. No significant large-scale topological patterns can be observed from these networks. Most such networks are mixed with circuits and branches. Among them, A2 and B2 are more circuit-like networks, where most arterials are located on circuits and they merge into a dominant web block. This is corroborated by the fact that both networks have higher measures of webness than their measures of treeness. On the other hand, arterials in Networks C2 and D2 are mostly connected as merged or scattered trees. Star patterns have been observed in these two networks.

The remaining four asymmetric networks A3, B3, C3, and D3 all display significant hub-and-spoke patterns. Each of these networks has the measure of treeness equal to 1 and is topologically characterized by arterials of higher levels connecting hubs and roads of those of lower levels connecting between hubs and terminals.

The measure of "beltness" indicates the significance of a beltway in the whole arterial system of a road network. Table 2 presents the measures of "beltness" for the six beltway networks. Both an outer beltway and an inner beltway are identified according to our definition in four out of six beltway cities, including networks $A 1$,

Table 2 Results of Proposed Structural Measures

\begin{tabular}{llllll}
\hline Topology & $\begin{array}{l}H \\
(\text { entropy })\end{array}$ & $\begin{array}{l}\phi_{\text {circuit }} \\
\left(\phi_{\text {ring }} / \phi_{\text {web }}\right) \\
\text { circuitness } \\
\text { (ringness/webness) }\end{array}$ & $\begin{array}{l}\phi_{\text {belt }}^{1} / \phi_{\text {belt }}^{2} \\
\text { (beltness) }\end{array}$ & $\begin{array}{l}\phi_{\text {tree }} \\
\text { (treeness) }\end{array}$ & $\begin{array}{l}Y \\
\text { (discontinuity) }\end{array}$ \\
\hline A0 & 0.00 & $\mathrm{NA}$ & $\mathrm{NA}$ & $\mathrm{NA}$ & 0.0000 \\
$\mathrm{~A} 1$ & 1.32 & $0.83(0.00 / 0.83)$ & $0.42 / 0.17$ & 0.17 & 0.0005 \\
$\mathrm{~A} 2$ & 1.31 & $0.78(0.00 / 0.78)$ & $0.44 / 0.14$ & 0.22 & 0.0017 \\
A3 & 1.28 & $0.00(0.00 / 0.00)$ & $0.00 / 0.00$ & 1.00 & 0.0056 \\
B0 & 0.00 & $\mathrm{NA}$ & $\mathrm{NA}$ & $\mathrm{NA}$ & 0.0000 \\
B1 & 0.45 & $0.49(0.49 / 0.00)$ & $0.49 / 0.00$ & 0.51 & 0.0000 \\
B2 & 1.01 & $0.68(0.00 / 0.68)$ & $0.29 / 0.12$ & 0.32 & 0.0001 \\
B3 & 1.26 & $0.00(0.00 / 0.00)$ & $0.00 / 0.00$ & 1.00 & 0.0016 \\
C0 & 0.00 & $\mathrm{NA}$ & $\mathrm{NA}$ & $\mathrm{NA}$ & 0.0000 \\
C1 & 1.66 & $0.69(0.00 / 0.69)$ & $0.38 / 0.00$ & 0.31 & 0.0270 \\
C2 & 0.99 & $0.32(0.04 / 0.28)$ & $0.00 / 0.00$ & 0.67 & 0.0144 \\
C3 & 1.88 & $0.00(0.00 / 0.00)$ & $0.00 / 0.00$ & 1.00 & 0.0342 \\
D0 & 0.00 & $\mathrm{NA}$ & $\mathrm{NA}$ & $\mathrm{NA}$ & 0.0000 \\
D1 & 0.68 & $0.77(0.00 / 0.77)$ & $0.36 / 0.19$ & 0.23 & 0.0117 \\
D2 & 0.54 & $0.00(0.00 / 0.00)$ & $0.00 / 0.00$ & 1.00 & 0.0162 \\
D3 & 1.43 & $0.00(0.00 / 0.00)$ & $0.00 / 0.00$ & 1.00 & 0.0324 \\
\hline
\end{tabular}

*The measures $\phi_{\text {belt }}^{1}$ and $\phi_{\text {belt }}^{2}$ indicate the beltness of the outer beltway and that of the inner beltway, respectively. The measure of beltness is equal to 0.00 when no beltway is detected. 
A2, B2, and D1, and so both measures of $\phi_{\text {belt }}^{1}$ and $\phi_{\text {belt }}^{2}$ are presented in Table 2. As can be seen, network B1 has the most significant beltway with the beltness equal to 0.49 , and it is the only beltway network that contains a ring block instead of a web block. Thus, its measure of "beltness" is equal to its "ringness" measure. The beltways of the other five beltway networks are identified as the envelopes of their dominant web blocks, and their measures of "beltness" are smaller than their "webness" measures.

The heterogeneity of test networks can be compared using their entropy measures. As can be seen in Table 2, networks A3, B3, C3, and D3 have relatively high entropies, indicating that hub-and-spoke structures are more differentiated than others. These networks also have higher measures of discontinuity, compared with the beltway networks. This can be explained by the fact that travelers have to access main roads that connect hubs via branches of different hierarchies in a huband-spoke, while they can access a beltway easier and usually travel continuously through roads of the same level on a beltway. In fact, the measures of entropy $(H)$ and discontinuity $(Y$ ) shown in Table 2 are highly correlated $(0.623)$, indicating that travelers experience higher discontinuity in a more heterogeneous network.

\section{Conclusions}

The structure of spatial networks and the measurement of their topologic, geometric, and large-scale statistic attributes are topics that deserve attention. Network scientists, geographers, transportation researchers, and urban planners have developed a variety of structural measures over half a century. This exploratory study arose from our ongoing investigation into the temporal development of road networks in which we observed that large-scale structural order, such as road hierarchies and connection patterns, emerged and were reinforced through time, and the flow patterns on the networks changed accordingly. No precise definitions and measurement of these structural attributes, however, have been found in the literature, especially for road networks. If these attributes can be assessed in a standard way, the temporal change of a network could then be traced down quantitatively, which provides a useful tool for geographers, network analysts, and transportation researchers to examine the evolution of networks. Moreover, statistical analysis of differentiated road networks across regions could disclose the correlation between the efficiency of transportation (such as mobility, accessibility, and safety) and structural attributes, with other factors controlled, thus providing guidance to transportation planners regarding how network designs affect efficiency in the long run. This study proposed three new measures that supplement existing measures for transportation networks, specifically examining the structure of urban road networks. These measures are heterogeneity (entropy), connection pattern, and continuity, respectively.

Instead of measuring the heterogeneity of a network based on power-law distribution as many contemporary network analyses do, we argue that a road network 
is link-centric and proposed a link-based measure of entropy to examine the heterogeneity of the network with regard to the differentiated function or performance of urban roads. Depending on the criteria we use to classify links, the measure of entropy has a variety of applications. Transportation planners always face a choice to build arterial roads for more long-distance mobility or to build collector roads to improve local access. An entropy measure based on functional classes of roads indicates the balance between the two functionalities, and could provide a benchmark for a more balanced design of network. An entropy measure on the basis of classified road conditions such as speed and LOS is a good indicator for engineers to distribute traffic flows more efficiently on a network. A particular distribution of links could be devised using entropy maximization techniques based on a sampling of roads in a network, which enables more empirical applications of this measure.

This study defines typical connection patterns and provides a standard way to algorithmically identify predefined patterns and to measure their respective significance in a network of arterials (highways). Previous studies on geometric patterns of spatial networks either examine only simplified patterns in idealized networks or recognize patterns manually. This method realizes automatic recognition of predefined geometric patterns, which is less costly, more accurate, reproducible, and not limited to simple networks. This method also makes it possible to conduct large-scale statistical analyses of connection patterns among a batch of networks, as computers are much faster in following programmed instructions and performing mathematical calculations. Compared with the aforementioned beltway study conducted in the 1980s, a similar statistical analysis can now be conducted in hundreds of networks within much less time. Note that, although exemplified by idealized networks, our algorithm can be applied to any digitized network coded in link-node structure. In addition, the proposed measures of connection patterns can be used to provide common yardsticks to compare connection patterns in different networks, as well as to precisely trace the structural change of networks over time.

The measure of contiguity provides a way to evaluate the quality of a road network from the travelers' perspective. On the basis of previous analyses of accessibility and interconnectivity, this measure considers both the attributes of heterogeneity and connectivity and the aggregate travel behavior (traffic flow) on the network. Based on the assumption that inconvenience is associated with transferring between different levels of roads, this measure can be extended to account for many "discontinuous" factors, such as the delay at traffic signals or ramp meters, the toll paid entering a toll road, merging, turns, etc. Developed based on transportation planning models, this measure could act as a guideline to transportation planners toward a better design of network structure. Together with the measures of connection patterns, the measures of desired network properties such as the measure of continuity could become a useful guide for urban planners in the design of collective patterns of urban roads. This measure has the flexibility to account for the discontinuity when transferring between different modes, as well as different levels 
of discontinuity perceived by different user classes, by introducing more sophisticated travel demand models.

\section{References}

Albert, R., H. Jeong, and A. L. Barabasi. (1999). "Diameter of the World-wide Web." Nature $401,130-31$.

Balch, T. (2000). "Hierarchic Social Entropy: An Information Theoretic Measure of Robot Team Diversity." Autonomous Robots 8, 209-38.

Barabasi, A. (2002). Linked: The New Science of Networks. Cambridge, MA: Perseus Publication.

Barabasi, A., and E. Bonabeau. (2003). "Scale-Free Networks." Scientific American 288(5), 50-59.

Ben-Naim, E., H. Frauenfelder, and Z. Toroczkai. (2004). Complex Networks. Berlin, Germany: Springer.

Bryan, D. L., and M. E. O'Kelly. (1999). "Hub-and-Spoke Networks in Air Transportation: An Analytical Review." Journal of Regional Science 39(2), 275-95.

Dill, J. (2004). "Measuring Network Connectivity for Bicycling and Walking." In 84th Annual Meeting of the Transportation Research Board. Paper 04-001550, Washington, DC.

Federal Highway Administration, U.S. Department of Transportation. (1997). Flexibility in Highway Design. Washington, DC: Author.

Garrison, W. L. (1960). "Connectivity of the Interstate Highway System." Regional Science Association, Papers and Proceedings 6, 121-37.

Garrison, W. L., and D. F. Marble. (1962). "The Structure of Transportation Networks." U.S. Army Transportation Command, Technical Report 62-II, 73-88.

Gauthier, H. L. (1966). "Highway Development and Urban Growth in Sao Paulo, Brazil: A Network Analysis." Ph.D. Dissertation, Northwestern University, Evanston, IL.

Gibbons, A. (1985). Algorithmic Graph Theory. Cambridge, UK: Cambridge University Press.

Hanson, S. (1986). The Geography of Urban Transportation. New York: Guilford.

Hargett, P., and J. C. Chorley. (1969). Network Analysis in Geography. London: Butler \& Tanner Ltd.

Jeong, H., B. Gombor, R. Albert, Z. N. Oltwai, and A. L. Barabasi. (2000). "The Large-Scale Organization of Metabolic Networks." Nature 407, 651-54.

Kansky, K. (1963). "Structure of Transportation Networks: Relationships between Network Geometry and Regional Characteristics." Research paper 84, Department of Geography, University of Chicago, Chicago.

King, L. (1985). Central Place Theory. London: Sage.

Lee, K., and H. Lee. (1998). "A New Algorithm for Graph-Theoretic Nodal Accessibility Measurement." Geographical Analysis 30, 1-14.

Levinson, D. (2002). Financing Transportation Networks. Northampton, UK: Edward Elgar Publishing Inc.

Levinson, D., and B. Yerra. (2006). "Self Organization of Surface Transportation Networks." Transportation Science 40(2), 179-88.

Liljeros, F., C. R. Edling, L. A. N. Amaral, H. E. Stanley, and Y. Aberg. (2001). "The Web of Human Sexual Contacts." Nature 411, 907-08. 
Marshall, S. (2005). Streets and Patterns. New York: Spon Press.

Minnesota Department of Transportation. (2005). Official Website: http://www.dot.state.mn. us/tda/html/faq.html

Mohring, H. (1961). "Land Values and the Measurement of Highway Benefits." Journal of Political Economy 79, 236-49.

Morrison, S. A., and C. Winston. (1989). "Airline Deregulation and Public Policy." Science $245,707$.

Newell, G. F. (1980). Traffic Flow on Transportation Networks. Cambridge, MA: MIT Press.

Newman, M. (2003). "The Structure and Function of Complex Networks." SIAM Review 45, $167-256$.

Ortuzar, J. D., and L. G. Willumsen. (2001). Modeling Transport. New York: Wiley.

Payne-Maxie Consultants. (1980). "The Land Use and Urban Development Impacts of Beltways." Final Report DOT-OS-90079, U.S. Department of Transportation and Department of Housing and Urban Development. Washington, DC: Payne-Maxie Consultants.

Price, D. (1965). "Networks of Scientific Papers." Science 149, 510-15.

Rietveld, P. (1995). "Some Notes on Interconnectivity in Transport Networks." In Overcoming Isolation: Information and Transportation Networks in Development Strategies for Peripheral Regions, 18-28, edited by H. Cocossis and P. Nijkamp. Berlin, Germany: Springer.

Rietveld, P. (1997). "Policy Aspects of Interconnectivity in Networks." In Networks in Transport and Communication: A Policy Approach, 177-92, edited by C. Capriani and P. Rietveld. Aldershot, UK: Avebury.

Rodrigue, J. (2004). Transport Geography on the Web. http://people.hofstra.edu/geotrans Shannon, C. E. (1948). "A Mathematical Theory of Communication." Bell System Technical Journal 27, 379-423, 623-56.

Sole, R. V., and S. Valverde. (2004). "Information Theory of Complex Networks: On Evolution and Architectural Constraints." Lecture Notes Physics 650, 189-207.

Taaffe, E., H. Gauthier, and M. O'Kelly. (1996). Geography of Transportation. Engelwood Cliffs, NJ: Prentice-Hall.

Taylor, D. (1995). Hub and Spoke Networks in Truckload Trucking: Configuration, Testing, and Operational Concerns. Logistics and Transportation 31, 209-37.

Trusina, A., S. Maslov, P. Minnhagen, and K. Sneppen. (2004). "Hierarchy Measures in Complex Networks." Physics Review Letter 92, 178702.

Vaughan, R. (1987). Urban Spatial Traffic Patterns. London: Pion Limited.

Yerra, B., and D. Levinson. (2005). "The Emergence of Hierarchy in Transportation Networks." Annals of Regional Science 39(3), 541-53.

Zhang, L. (2006). Ph.D. Dissertation: Search, Information, Learning, and Knowledge in Travel Decision-Making: A Positive Approach for Travel Behavior and Demand Analysis. University of Minnesota, Minneapolis, MN. 